\title{
E-NEGOTIATION: CAN ARTIFICIAL INTELLIGENCE NEGOTIATE BETTER DEALS?
}

\author{
Gordana Dobrijević, \\ Filip Đoković \\ Faculty of Business, \\ Singidunum University, \\ Belgrade, Serbia
}

\begin{abstract}
:
Artificial intelligence can perform many different tasks in negotiation, reducing time and effort on the part of human negotiators. Today there is an increasing number of negotiation support systems and automated negotiating agents, that can assist human negotiators before and during the process. They can serve as simulators and training tools, but also conduct negotiations more or less autonomously. The aim of this paper is to review different forms of electronic negotiations and current issues in this field.
\end{abstract}

Keywords:

e-negotiation, negotiation support systems, automated negotiation, automated negotiation agents.

\section{Correspondence:}

Gordana Dobrijević

e-mail:

gdobrijevic@singidunum.ac.rs

\section{INTRODUCTION}

Negotiation is an interaction between parties who are trying to agree on their opposing interests and find a mutually beneficial outcome. Although negotiation is normally thought of as a human activity, artificial intelligence plays an important part in contemporary business negotiation. Negotiation support systems (NSSs) and software agents were developed not only to help people with repetitive and tiresome tasks, but also to help them overcome unchecked emotions and some common cognitive errors and biases, such as using shortcuts in decision making, selective perception, and framing (making decisions based on positive or negative presentation of options). They can be used to train and/or support human negotiators, to negotiate instead of people, or act as brokers between companies [1]. Employing software agents in negotiations can generate better results, reduce costs, and cause fewer conflicts [2]. 


\section{ELECTRONIC NEGOTIATION}

Electronic negotiation (e-negotiation) can be defined as negotiating via electronic means. Different electronic devices can be used in the process, from simple chat or e-mail, to online negotiation platforms and autonomous software agents. E-negotiation can help human negotiators focus on the subject of negotiation, rather than personal issues [3].

\section{E-mail negotiations}

Negotiation over e-mail is ubiquitous in contemporary business. It can save time and costs, but also damage trust and obstruct communication, due to the absence of nonverbal cues. Especially when communicating crossculturally, it is hard to convey the tone of conversation and respect the norms of polite behaviour of a different culture [4]. The research of Rosette, Brett, Barsness, \& Lytle [5] on cross-cultural negotiation showed that, when negotiating over e-mail, people give less favourable opening offers then in traditional negotiation. Typically, when negotiating via e-mail people become more antagonistic and impasses happen more often. Due to a faulty sense of anonymity, people become more hostile and give more ultimatums. Negotiators need more time and effort to build relationship with another party in online negotiations. Different studies have demonstrated that communication media influence the negotiation process and results [6].

Another important issue in cross-cultural e-negotiation happens during the pre-negotiation phase. The first impressions and agreeing on the communication channel to be used in the process affect the relationship and the conclusion of negotiation [7]. Sokolova and Lapalme [8] discovered that in successful negotiations parties tended to use more positive expressions at the start. This shows us that the outcome can be predicted at the very beginning of negotiation, by analyzing the words the parties use.

\section{Negotiation Support Systems}

The Internet facilitated the creation and use of decision and negotiation support systems. The group decision support systems (GDSS) were developed to facilitate decision making while avoiding "false consensus", that way reaching a politically achievable agreement that will actually be implemented. The two main benefits of the GDSS are achieving procedural justice and avoiding groupthink [9].
Participants' perception of fairness encourages them to behave cooperatively and engage in attaining group goals. This is especially important in negotiations between different organizations. Groupthink happens when a group exerts extensive pressure on an individual to conform to others' opinions [10]. Groups can then easily fall into the trap of ignoring alternatives, due to psychological pressure for consensus. Other important characteristics of GDSSs are anonymity, face-saving features, and productivity, the last being achieved by generating small-scope agreements at regular intervals. In some forced private-public partnerships, GDSSs enable the negotiating parties to create additional value together, and to go beyond and above their own objectives [9].

The earliest negotiation support systems (NSSs) were created in the 1980s [11], in order to aid human negotiators during different stages of the negotiation process, with the tasks like understanding the issues, deciding on reservation price, choosing the preferences and acceptable alternatives [12]. NSSs can reduce unreasonable behaviour of human negotiators, speed up the process, and create higher gains for both (all) parties included [13]. However, human negotiators still have to administer the process themselves [2].

Some well-known online NSSs are Pocket Negotiator [14], Invite [15], and Smartsettle [16]. They can be employed as negotiation simulators, training tools, research tools, and support during the process. Some of their versions can be used in complex multi-party multi-issue situations.

Pocket Negotiator will be used as an illustration of the use of NNSs. In the preparation phase, the user chooses the domain, i.e. real estate, energy, water management or jobs and then the strategy to be used (e.g. Pareto Optimal Concede, Nice Mover, Tough Negotiator, etc.). The user also defines the position and preferences of the opposing party. In the exploration phase, the negotiators get to know each other and build their profiles. In both preparation and exploration phases users identify their interests and preferences. The system allows qualitative preference identification, based on principled negotiation, created by Harvard Program on Negotiation. It supports human negotiators in identifying their preferences, estimating the preferences of the other party, during the bidding (bargaining) stage and closing the deal. In determining preferences, the user is offered advice and given an opportunity to change some items (Figure 1). In the bidding phase the negotiating parties offer bids to each other and the user gets advice related to when to accept a bid from the other side [17]. 


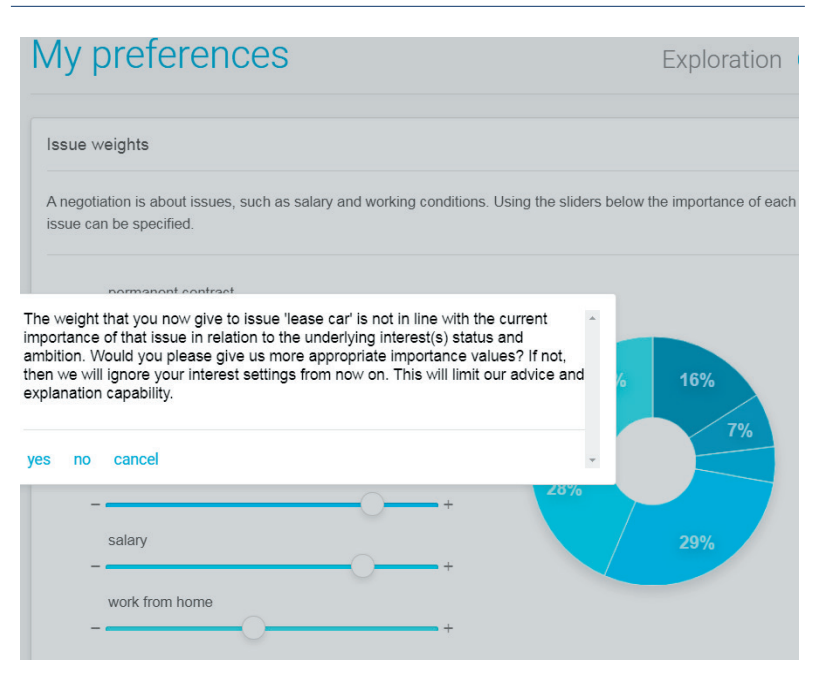

Figure 1. Pocket Negotiator software, personal preferences (exploration phase) [14]

NSSs are employed even in dealing with complicated international natural resource conflicts, e.g. demarcating the Caspian Sea and its implications to the gas, oil, and water exploitation by Russia, Iran, Kazakhstan, Azerbaijan and Turkmenistan. The Caspian Sea Negotiation Support System took into consideration not only established rules for dividing the Sea by area, but also fair distribution of natural resources between interested parties [18].

Game theory is one of the mechanisms for developing NSSs. In game theory the parties choose whether to behave cooperatively or competitively, i.e. to care only about their own interests or to cooperate with the other side to achieve higher mutual gains. Still, in real life situations this joint reward is not always obvious, due to insufficient information. That is why Zandi and Tavana [3] applied fuzzy sets to manage this vagueness. Fuzzy logic mimics human decision making and can include all values between 0 and 1 .

\section{Automated Negotiation}

Automated negotiation is a process in which autonomous agents interact to reach an agreement. Software agents can conduct negotiations on behalf of humans. The Diplomat Agent, the Colored Trails Agent, the Guessing Heuristic Agent, and the Cliff-Edge Agent are some of the well-known automated agents [19].

Their usage usually generates lower costs, fewer conflicts and higher mutual gains. However, some pieces of research had different findings. For example, comparing negotiations between humans and between automated agents, Filzmoser [20] analyzed three aspects of negotiation outcome: the number of agreements reached, individual negotiators' efficiency, and fairness. The study showed that agents reached higher outcomes, but they were less fair and with lower mutual gain than human negotiators.

The most noticeable difference between automated negotiations and traditional negotiations with human participants is the number of interactions. While human negotiators usually make just several offers and counteroffers, automated agents interchange hundreds and even thousands of bids in a minute [21].

Various methodologies have been used in agent design, e.g. Gaia [22], MESSAGE [23], Aspecs [24], and KEMNAD (A Knowledge Engineering Methodology for Negotiating Agent Development). KEMNAD is made of a generic knowledge model and different standardized templates that can be used repeatedly. Diverse negotiation models are created by incorporating different templates [25].

A negotiation process between two automated agents ( $A$ and $B$ ) is presented on Figure 2. The setting consists of the agents, the negotiation scenario, and the protocol. An agent can perform only the actions prescribed by the protocol and the scenario. Preference profile shows preferred and feasible outcomes for each party. The negotiation domain defines the sequence of possible outcomes. To come to an agreement, agents must accept a particular value for each issue. Negotiation time is restricted, so that the parties have to accept an offer instead of indefinitely waiting for a better deal [21].

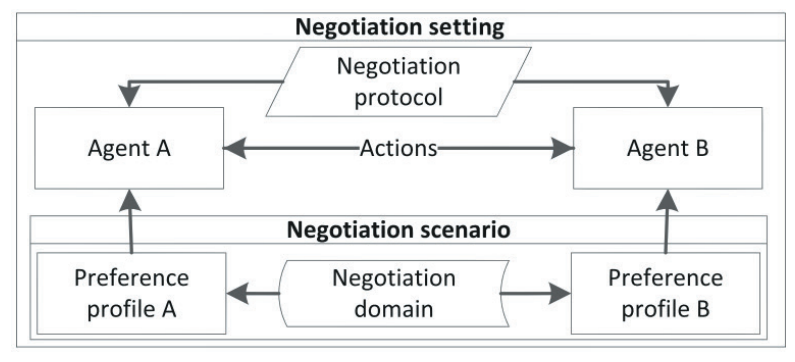

Figure 2. Overview of wthe defining elements of an automated bilateral negotiation [21]

Aydoğan et al. [26] describe two negotiation protocols, called Stacked Alternating Offers Protocol (SAOP) and Alternating Multiple Offers Protocol (AMOP). In both of them, automated agents take turns in making or accepting the bids. The manner of exchange is determined before the negotiation starts. SAOP permits agents to make an offer, accept the latest offer or walk away. On the contrary, AMOP does not allow walking away. 
It has a bidding and a voting stage. In both protocols (apart from the walking away option in SAOP), negotiation ends when the agents come to an agreement or the deadline is past. In their empirical research, SAOP was better than AMOP with the same kind of agents.

One of the focal points of agent development is the so-called opponent modeling, i.e. learning about the opponent. Agents get information about the other party from their bidding sequence and communication in general [27]. The agent has to assess the other party's preferences and possible strategy. The opponent modeling techniques can be applied to various negotiation protocols. Different protocols may vary in the domain configuration, accessible information, the number of agents, interaction between issues, etc. [21].

Different learning methods can be used in automated negotiation. Bayesian Learning is the most famous probabilistic approach in opponent modeling. It is normally applied to determine the most probable hypothesis out of a given set of hypotheses. Usually a finite set is assumed [21]. One of the latest is RLBOA, a modular reinforcement learning framework. It is based on trialand-error learning, where software gets rewards and penalties for its activities [28].

Automated negotiating agents are developed for specific use in particular scenarios, which makes it difficult to compare their effectiveness. The Automated Negotiating Agents Competition (ANAC) is an annual event (since 2010) for evaluation and comparison of automated agents [29]. The competition is carried out within the GENIUS environment (General Environment for Negotiation with Intelligent multi-purpose Usage Simulation), developed by Lin and Kraus [30]. The competition challenges researchers and developers to create automated agents to compete in scenarios with limited information about the opposing party. This competition brought about important advances in agent development.

Because human-agent and human-human negotiation are very different, a separate Human-Agent Negotiation League was established within ANAC in 2017 [27]. This contest is carried out on the IAGO Negotiation platform, designed by Mell and Gratch [31]. IAGO (Figure 3) produces a graphical user interface for human negotiators. IAGO's characteristics enable agents to simulate human communication, such as using different communication media. Several automated agents that participate in this competition use emotion to influence the other party, like positive emotions for building rapport, or negative to get concessions.
Some of them also lie and deceive the other party. Some automated agents can be programmed to show some other human characteristics, such as warmth, or to behave irrationally or vindictively [32]. Then it becomes hard for human negotiators to tell if their opposing party is a human or a computer. However, when they know who they negotiate with, people behave differently towards agents and people. The research of de Melo, Marsella, and Gratch [33] showed that people give better offers to humans than to agents who negotiate instead of humans.

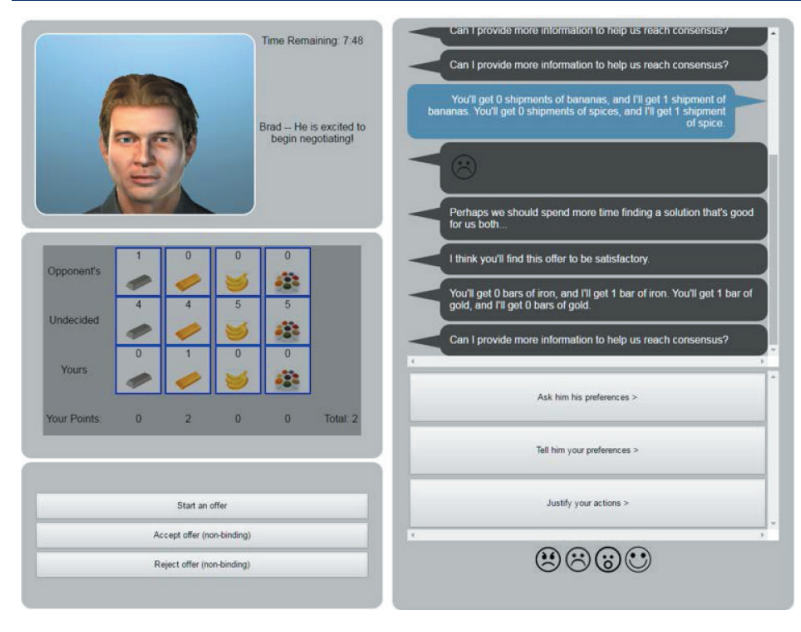

Figure 3. IAGO Research Platform, Client view [27]

\section{SOME POTENTIAL PROBLEMS IN E-NEGOTIATIONS}

One of the challenges of e-negotiations is creating a fully autonomous agent, capable of negotiating in any setting, in any area. Contemporary agents are mostly developed with specific type of problems in mind and for specific domains. Truly autonomous agents should be able to work with limited information, as is the case with face-to-face negotiation between humans.

Automation is closely related to another challenge, which is expressing preferences by human users. Sometimes people are unwilling or unable to state their preferences when using a NSS, because there are simply too many questions to go through. This issue can be solved by the system requesting preferences gradually, during the course of the negotiation process, rather than all at the beginning. Another issue is trust, which is hard to build even in face-to-face negotiation between humans [34]. There is also the problem of accidentally sending meta-data to the other party, which could contain confidential information. 


\section{CONCLUSION}

Artificial intelligence can help human negotiators with many elements of negotiation process, such as the analysis of preferences and viable alternatives. It can also serve as a training tool or conduct negotiation on behalf of humans. The synergy between a man and a machine can help humans with laborious tasks, while avoiding cognitive errors and unchecked emotions.

There are still many technical matters that have to be resolved before artificial intelligence can be universally used in real-life negotiations. Successful software agents should be able to negotiate with different people in different negotiation settings. Trust and emotions are still key factors when humans are involved in negotiations. Automated agents can be programmed to behave like human beings, to show anger and kindness. But do we really want that?

\section{REFERENCES}

[1] A. van Wissena, Y. Galb, B.A. Kamphorstd, and M.V. Dignume, "Human-agent teamwork in dynamic environments," Computers in Human Behavior, vol. 28, No. 1, pp. 23-33, 2012. doi:10.1016/j. chb.2011.08.006

[2] Y. Yang, S. Singhal, and Y. Xu, "Alternate strategies for win-win seeking agent in agent-human negotiations," Journal of Management Information Systems [serial online], vol. 29, No. 3, pp. 223-256, 2012. Retrieved January 31, 2014, from Business Source Premier, Ipswich, MA.

[3] F. Zandi and M. Tavana, "A fuzzy e-negotiation support system for inter-firm collaborative product development," International Journal of Computer Integrated Manufacturing, vol. 25, No. 8, pp. 671688, 2012.

[4] Pon Harvard, "The pitfalls of negotiations over email."Retrieved December 11, 2019, from https:// www.pon.harvard.edu/daily/conflict-resolution/ email-more-cons-than-pros/

[5] A.S. Rosette, J.M. Brett, Z. Barsness, and A.L. Lytle, "When cultures clash electronically: The impact of email and social norms on negotiation behavior and outcomes," Journal of Cross-Cultural Psychology, vol. 20, No. 10, pp. 1-16, 2011. Retrieved December 11, 2019, from https://www.academia. edu/15507677/When_Cultures_Clash_Electronically_The_Impact_of_Email_and_Social_Norms_ on_Negotiation_Behavior_and_Outcomes. doi: 10.1177/0022022111407190.
[6] R.J. Lewicki, D.M. Saunders, and B. Barry, Negotiation, international edition. Singapore: McGrawHill, 2010.

[7] P. Kesting and R. Smolinski R. "Starting from scratch: A multi-stage analysis of remote e-negotiations." In Proceedings of the Group Decision and Negotiation conference. Stockholm, Sweden: University of Stockholm, 2013.

[8] M. Sokolova and G. Lapalme, "How much do we say? Using informativeness of negotiation text records for early prediction of negotiation outcomes," Group Decision and Negotiation, vol. 21, pp. 363379, 2010.

[9] C. Eden and F. Ackermann, “Joined-up' policymaking: Group decision and negotiation practice." In Proceedings of the Group Decision and Negotiation conference. Stockholm, Sweden: University of Stockholm, 2013. Retrieved December 15, 2019, from https://www.researchgate.net/ publication/266149403_'Joined-Up'_Policy-Making_Group_Decision_and_Negotiation_Practice. doi: 10.1007/s10726-013-9375-1

[10] S. Robbins and M. Coulter, Management, 13th ed. Harlow: Pearson Education Limited, 2016.

[11] L.H. Lim and I. Benbasat, "A theoretical perspective of negotiation support systems," Journal of Management Information Systems, vol. 9. No. 3, pp. 27-44, 1992.

[12] G. Dobrijević and J. Đorđević Boljanović, "Negotiation goes high tech: Can you negotiate with a machine?" In Proceedings of the International Scientific Conference on ICT and E-Business Related Research SINTEZA 2014. Belgrade: Singidunum University.

[13] R. Vahidov, G.E. Kersten, "Design of software agentpopulated electronic negotiation system and evaluation of human-to-agent negotiations", Interneg Research Papers INR09/12, 2012. InterNeg Research Center, John Molson School of Business, Concordia University. Retrieved December 2, 2019, from http:// interneg.concordia.ca/index.php?id=paper.

[14] TU Delft (n.d.). Pocket Negotiator. Retrieved September 8, 2019, from http://ii.tudelft.nl:8080/PocketNegotiator/index.jsp.

[15] Invite Negotiation Systems (n.d). Retrieved December 13, 2019, from http://invite.concordia.ca/.

[16] Smartsettle, Beyond Win-Win (n.d.). Retrieved December 1, 2019, from: https://smartsettle.com.

[17] C. Jonker, R. Aydoğan, T. Baarslag, J. Broekens1, C. Detweiler, K. Hindriks, A. Huldtgren, and W. Pasman, "An introduction to the Pocket Negotiator: A general purpose negotiation support system," in N. Criado Pacheco, C. Carrascosa, N. Osman N., \& V. Julián Inglada, Eds., Multi-Agent Systems and 
Agreement Technologies. EUMAS 2016, AT 2016. Lecture Notes in Computer Science, vol. 10207. Cham: Springer. doi: 10.1007/978-3-319-59294-7_2 2017

[18] K. Madani, O.M. Rouhani, A. Mirchi, and S. Gholizadeh, "A negotiation support system for resolving an international trans-boundary natural resource conflict," Environmental Modelling \& Software, vol. 51, pp. 240-249, 2014.

[19] R. Lin, S. Kraus, "Can automated agents proficiently negotiate with humans?" Communications of the ACM [serial online], vol. 53, No. 1, pp.7888, January 2010. Available from: Business Source Premier, Ipswich, MA, accessed January 31, 2014. doi:10.1145/1629175.1629199

[20] M. Filzmoser, “Automated vs. Human Negotiation," International Journal of Artificial Intelligence, vol. 4, No. 10, pp. 64-77, 2010.

[21] T. Baarslag, M. Hendrikx, K. Hindriks, and C. Jonker, "Learning about the opponent in automated bilateral negotiation: a comprehensive survey of opponent modelling technique," Autonomous Agents and Multi-Agent Systems, vol. 30, No. 5, pp. 849898, 2016. doi: 10.1007/s10458-015-9309-1

[22] M. Wooldridge, N.R. Jennings, and D. Kinny, “The Gaia methodology for agent-oriented analysis and design", Journal of Autonomous Agents and MultiAgent Systems, vol. 3, No.3, pp. 285-312, 2000.

[23] G. Caire, F. Leal, P. Chainho, R. Evans, F. Garijo, J. Gomez, J. Pavon, P. Kearney, J. Stark, and P. Massonet, "Agent oriented analysis using MESSAGE/ UML", in Proceedings of the Second International Workshop on Agent-Oriented Software Engineering, Montreal, Canada, pp. 101-108, 2001.

[24] M. Cossentino, and N. Gaud, V. Hilaire, S. Galland, and A. Koukam, "Aspecs: An agent-oriented software process for engineering complex systems", Autonomous Agents and Multi-Agent Systems, vol. 20, No. 2, pp.260-304, 2010.

[25] X. Luo, C. Miao, N. Jennings, M. He, Z. Shen, and M. Zhang, "KEMNAD: A knowledge engineering methodology for negotiating agent development", Computational Intelligence, vol. 28, No. 1, pp. 51-105, February 2012

[26] R. Aydoğan, D. Festen, K.V. Hindriks, and C.M. Jonker, "Alternating offers protocols for multilateral negotiation," in K. Fujita, Q. Bai, T. Ito, M. Zhang, F. Ren, R. Aydoğan, and R. Hadfi, Eds, Modern Approaches to Agent-based Complex Automated Negotiation, pp. 153-167, 2017. Cham: Springer International Publishing AG. doi: 10.1007/978-3319-51563-2_10

[27] J. Mell, J. Gratch, T. Baarslag, R. Aydoğan, R. and C. Jonker, "Results of the first annual Human-Agent League of the Automated Negotiating Agents
Competition," in Proceedings of the International Conference on Intelligent Virtual Agents (IVA '18). Sydney, NSW, Australia: DBLP Computer Science Bibliography, 2018. doi: 10.1145/3267851.3267907

[28] J. Bakker, A. Hammond, D. Bloembergen, and T. Baarslag, "RLBOA: A modular reinforcement learning framework for autonomous negotiating agents," in Proceedings of the 18th International Conference on Autonomous Agents and Multiagent Systems (AAMAS 2019), N. Agmon, M. E. Taylor, E. Elkind, and M. Veloso, Eds., Montreal, Canada: IFAAMAS, 2019.

[29] C. Jonker, R. Aydoğan, T. Baarslag, K. Fujita, T. Ito, and K. Hindiks, "Automated Negotiating Agents Competition (ANAC)," in Proceedings of the Thirty-First AAAI Conference on Artificial Intelligence (AAAI-17), San Francisco, CA, pp. 5070-5072, 2017.

[30] R. Lin and S. Kraus, "From research to practice: Automated negotiations with people," in A. Krüger and T. Kuflik, Eds., Ubiquitous Display Environments, pp. 195-212. Berlin Heidelberg: SpringerVerlag, 2012.

[31] J. Mell and J. Gratch, "IAGO: interactive arbitration guide online," in Proceedings of the 2016 International Conference on Autonomous Agents \& Multiagent Systems. Singapore: International Foundation for Autonomous Agents and Multiagent Systems, 2016.

[32] P. Prajod, M. Al Owayyed, T. Rietveld, J. van der Steeg, and J. Broekens, "The effect of virtual agent warmth on human-agent negotiation," in Proceedings of the 18th International Conference on Autonomous Agents and Multiagent Systems (AAMAS 2019), N. Agmon, M. E. Taylor, E. Elkind, \& M. Veloso (Eds.). Montreal, Canada: IFAAMAS, 2019.

[33] C.M. de Melo, S. Marsella, and J. Gratch, "Do as I say, not as I do: Challenges in delegating decisions to automated agents," in Proceedings of the 2016 International Conference on Autonomous Agents \& Multiagent Systems. Singapore: International Foundation for Autonomous Agents and Multiagent Systems, 2016.

[34] T. Baarslag, M. Kaisers, E. Gerding, C. Jonker, and J. Gratch, "When will negotiation agents be able to represent us? The Challenges and Opportunities for Autonomous Negotiators," in Proceedings of the Twenty-Sixth International Joint Conference on Artificial Intelligence (IJCAI-17). Melbourne, Australia: DBLP Computer Science Bibliography, 2017. 